\title{
La Révolution aux barrières : l'incendie des barrières de l'octroi à Paris en juillet 1789
}

The Revolution of the Custom Posts: the Burning of the Custom Posts of the Octroi in July 1789

Momcilo Markovic

\section{(2) OpenEdition}

\section{Journals}

\section{Édition électronique}

URL : https://journals.openedition.org/ahrf/12765

DOI : $10.4000 / a h r f .12765$

ISSN : 1952-403X

Éditeur :

Armand Colin, Société des études robespierristes

Édition imprimée

Date de publication : 1 juin 2013

Pagination : $27-48$

ISBN : 978-2-9083-2789-2

ISSN : 0003-4436

\section{Référence électronique}

Momcilo Markovic, "La Révolution aux barrières : l'incendie des barrières de l'octroi à Paris en juillet 1789 », Annales historiques de la Révolution française [En ligne], 372 I avril-juin 2013, mis en ligne le 01 juin 2016, consulté le 01 juillet 2021. URL : http://journals.openedition.org/ahrf/12765 ; DOI : https://doi.org/10.4000/ahrf.12765 


\title{
LA RÉVOLUTION AUX BARRIÈRES : L'INCENDIE DES BARRIÈRES DE L'OCTROI À PARIS EN JUILLET 1789
}

Momcilo MARKOVIC

\begin{abstract}
En s'attaquant aux barrières parisiennes de l'octroi en juillet 1789, le peuple de Paris détériore le mur des Fermiers généraux, montrant son intention de supprimer les impôts aux portes de la ville. Plus qu'une émeute sans lendemain, il s'agit aussi pour la population d'affirmer qu'elle prend une place importante, ignorée jusque-là. L'assaut aux barrières est mené conjointement par le peuple et les principaux fraudeurs. En insistant sur la chute de la Bastille, les historiens n'ont-ils pas occulté l'importance de l'incendie des barrières qui, pourtant, permet que la forteresse soit prise ? Ce sont deux mouvements simultanés et parallèles qui voient le jour, mais se renforcent pour œuvrer vers le même but, celui d'exister sur la scène politique.
\end{abstract}

Mots-clés : Paris, mur des Fermiers généraux, barrières, foule, fraudeurs, incendie, espace public.

\section{L'incendie des barrières, une histoire négligée}

Si le 14 juillet 1789 a une valeur symbolique dans la mémoire des Français, l'attaque, le pillage, la destruction et l'incendie des nombreuses barrières de l'octroi qui ceinturaient Paris représentent un épisode moins connu. Cette péripétie est reléguée à un niveau moindre, comme si cette phase était la suite logique des tensions qui culminaient depuis le début du mois de juillet et dont l'aboutissement évident serait la prise de la Bastille, aventure qu'il est digne de raconter, car cette dernière marquerait le début 
du règne de la liberté. De fait, peu de lignes, peu d'explications ont été écrites sur cet événement qui, pourtant, précède la prise de la Bastille ${ }^{1}$.

Dans son livre monumental sur le faubourg Saint-Marcel, Haïm Burstin consacre plusieurs pages aux barrières et aux conséquences immédiates et ultérieures qu'elles eurent auprès de la population et du pouvoir. $\mathrm{Si}$ l'auteur examine plus attentivement ce qui se passe dans les quartiers qu'il étudie, il pointe du doigt un aspect essentiel : de manière quasi-simultanée, pendant que les barrières étaient assaillies, pillées et brûlées, se déroulait l'attaque contre la Bastille. Selon l'auteur, il y aurait eu deux mouvements de mobilisation, «l'un représentant le gros du courant insurrectionnel, l'autre [...] alimenté par des motifs particuliers de mécontentement $»^{2}$. Deux impulsions qui coexistent, mais qui n'œuvrent pas vers le même but. Il faut bien comprendre ce qu'écrit Haim Burstin qui, pour ainsi dire, dissocie ces deux rassemblements, et range le premier dans une catégorie hors-classe, à un échelon élevé, puisqu'il participe à une naissance politique, tandis que le deuxième est classé dans une typologie inférieure, mue par des intérêts catégoriels et non généraux.

Que pouvait ressentir le peuple de la capitale, sinon de l'incrédulité, voire de la colère en s'apercevant que sa ville devenait totalement emmurée et coupée par ces fameux bâtiments que Ledoux appelait les Propylées ? Barricadé de l'intérieur, enfermé uniquement pour des raisons fiscales, le peuple parisien ne pouvait que constater le gouffre financier que représentait la construction du mur des Fermiers généraux ${ }^{3}$. Le bâillonnement de la population continuait à un moment où le roi lui donnait la parole. Triste paradoxe qui, manifestement, ne pouvait que faire réagir le peuple de Paris.

(1) Pour une vision d'ensemble sur le mur des Fermiers généraux, Ephège FrÉMY, « L'enceinte de Paris construite par les fermiers généraux et la perception des droits d'octroi de la ville (1784-1791) », Bulletin de la Société de l'histoire de Paris et de l'Ile-de-France, 1912, 39ème année ; Jean-Marc PEYSSON, «Le mur d'enceinte des Fermiers-généraux et la fraude à la fin de l'Ancien Régime », Bulletin de la Société de l'histoire de Paris et de l'Ile-de-France, $109^{\mathrm{ème}}$ année, 1982, p. 225-240; Jean-Marc PEYSSON, Le mur d'enceinte des Fermiers-généraux : politique, économie, urbanisme, 2 vol., thèse de 3e cycle, Université Paris I, 1983. Sur l'incendie et le procès des incendiaires, Victor DE CLERCQ, «L'incendie des barrières de Paris en juillet 1789 et le procès des incendiaires », Bulletin de la Société de l'histoire de Paris et de l'Ile-de-France, 1938, t. 65, p. 31-48; Roger DiON, Histoire de la vigne et du vin en France, des origines au XIXe siècle, 1959, Paris, Flammarion, 1977 ; George RuDÉ, La foule dans la Révolution française, trad. fr. Paris, Maspero, 1982.

(2) Haim Burstin, Une Révolution à l'œuvre: le faubourg Saint-Marcel (1789-1794), Seyssel, Champ Vallon, 2005, p. 62.

(3) Archives nationales (désormais AN), Q ${ }^{1} 1101$, Clôture de Paris, mémoire de la commission ; celle-ci, chargée d'évaluer les dépenses du mur au début de l'année 1790, constatait que le montant des travaux s'élèverait à plus de 17 millions de livres. Dans les archives, à aucun moment l'appellation mur des Fermiers généraux n'apparaît ; on parle de clôture de Paris dans les documents officiels. J'ai cependant opté pour le premier vocable qui est toujours utilisé dans les ouvrages lorsqu'il désigne le mur et les limites de Paris. 
Les attaques aux barrières se superposent au mécontentement parisien, créant ainsi une dynamique, mélangeant et confrontant des catégories de population distinctes et diverses. La chute de la forteresse vole la vedette aux barrières et occulte presque cet événement négligé. Il faut redonner la juste place qui revient à la chute des barrières et montrer que les incidents du mois de juillet 1789 ne sont pas imputables uniquement à des fraudeurs, comme cela est encore écrit trop souvent. La complexité de la situation durant l'été, où les événements s'entrechoquent et s'enchevêtrent, trouble la vision et empêche de percevoir la réalité et l'importance de la «prise » des barrières. À bien lire les historiens, le mot « prise » n'est jamais accolé aux barrières. Les auteurs parlent de destruction, d'incendie, mais nullement de la «prise des barrières ». Et si la Bastille à prendre n'était pas uniquement celle que l'on croit être ? En d'autres termes, l'événement majeur de ce mois de juillet 1789 - la prise de la Bastille - doit être apprécié sous un autre angle, où la chute des barrières apporte un autre éclairage, autonome, et prend une place à part.

\section{Le Mur des Fermiers Généraux : un irrémédiable besoin fiscal}

Aujourd'hui, dans le paysage urbain de la capitale, il subsiste encore quelques-uns de ces édifices construits à la fin du XVIII ${ }^{\mathrm{e}}$ siècle par l'architecte Ledoux. Ne mentionnons que la rotonde de la Villette, bâtiment imposant, situé face au bassin de la Villette. Pour les autres constructions détruites, il nous reste des estampes, gravures et photographies des XIX ${ }^{\mathrm{e}}$ et $\mathrm{XX}^{\mathrm{e}}$ siècles $^{4}$. Bâtiments fastueux qui tranchaient avec l'objet même de leur fonction et qui, dès le début de leur construction en 1785, provoquèrent incompréhension et sarcasmes auprès d'une partie éclairée de la population parisienne. Ce qui est critiqué par la presse et les pamphlets demeure l'opulence de ces édifices et non l'enfermement de Paris. Il n'y a dans ces textes nulle remise en cause des taxes ${ }^{5}$.

D'une ville ouverte, Paris allait devenir en un temps record, entre 1785 et 1790, une métropole fermée. La capitale se trouva murée sur un périmètre long de 24 kilomètres, où la Ferme percevait à ses portes, à

(4) Les photographes Hyppolite Bayard et Charles Marville nous ont laissé de nombreuses photographies montrant les bâtiments de l'octroi qui n'étaient pas encore détruits avant l'annexion de 1860. Pour un aperçu de ces édifices disparus, on peut consulter Daniel RABREAU, Claude NicolasLedoux, l'architecture et les fastes du temps, Bordeaux, William Blake \& Co. /Art \& arts, 2000. Voir les dessins réalisés par Prieur où celui-ci représente à trois reprises les barrières dans Philippe DE CARBONNIÈRES, Prieur : les tableaux historiques de la Révolution, Collections du musée Carnavalet, Paris musées, 2006.

(5) Jean Marc PEYsson, art. cit., p. 230-235. 
ses barrières, des droits d'entrée. Le projet initial de boucler Paris revient à la Ferme. Cette dernière fournissait des avances à la royauté et percevait donc des impôts auprès de la population ${ }^{6}$. La fiscalité s'est alourdie depuis le XVII ${ }^{\mathrm{e}}$ siècle, faisant subir aux contribuables une pression de plus en plus forte. Les incidents concernant les impôts sont très nombreux au $\mathrm{XVIII}^{\mathrm{e}}$ siècle et peu de régions demeurent épargnées par les rébellions liées à la fiscalité, Paris connaissant une situation identique ${ }^{7}$. La Ferme persuade donc Louis XVI qui donne son consentement à l'édification d'une clôture afin d'empêcher la fraude, notamment sur les boissons (le vin et l'eau-de-vie), qui faisait perdre des entrées d'argent colossales ${ }^{8}$. Comment alors contrer les fraudeurs, et éviter les grosses pertes que subissait le Trésor royal ? L'idée est simple : il suffit tout bonnement de déplacer la modeste palissade en bois qui existe depuis de nombreuses années, enlever les bornes qui matérialisent les limites de la capitale et reculer ces dernières de plusieurs centaines de mètres vers l'extérieur, du côté des faubourgs, en les remplaçant par un mur de pierre, haut de plus de trois mètres, coupé par ces imposants bâtiments d'octroi, destinés à percevoir les taxes. Ainsi, les habitants vivant au dehors de la ville sont assujettis à la taille, alors que les Parisiens, exemptés de cet impôt, paient des droits sur les marchandises, aussi diverses que les matériaux de construction (plâtre, bois...), les produits alimentaires et les alcools (vin, eau-de-vie, cidre, poiré). Ces boissons restent évidemment moins chères hors barrière, le prix étant en moyenne trois fois moins élevé qu'à l'intérieur de Paris.

Pour s'atteler à ce projet grandiose, les Fermiers généraux font appel à l'architecte Ledoux ${ }^{9}$. Le tracé de la future enceinte était défini : la barrière devait être reculée de manière à enclore l'hôpital général de la Salpêtrière ; au sud, le mur englobait Vaugirard, le Champ de Mars et le Gros-Caillou. Sur la rive droite, la clôture cernait Picpus, reprenait une partie des quartiers de Belleville, de la Villette et des Porcherons, et se terminait à l'ouest par la Petite Pologne et Chaillot. Les travaux débutent rapidement dans la partie méridionale et, au début de l'année 1786, les bâtiments et le mur sont terminés et la perception est établie. Les tronçons sont mis en service à des

(6) Yves DURAND, Finances et mécénat, les fermiers généraux au XVIIIème siècle, Paris, Hachette, 1976, p 13.

(7) Jean NiCOLAS, La rébellion française, mouvements populaires et conscience sociale (1661-1789), Paris, Le Seuil, 2002 ; l'auteur, en recensant les violences durant cette période, fait apparaître que les révoltes et rébellions contre la fiscalité constituent plus de $39 \%$ de l'ensemble, p. 36.

(8) Les pertes financières, selon le célèbre chimiste et fermier général Lavoisier, avoisinent 48 millions de livres.

(9) Sur Ledoux, voir Daniel Rabreau, op. cit. ; Michel Gallet, Claude-Nicolas Ledoux, Paris, Picard, 1980. 
dates différentes sur la rive droite, en fonction des achats de terrain qui prennent du temps. Ainsi, durant l'année 1788, une première tranche est ouverte à l'ouest, en partant de la Seine jusqu'à la place du Roule. Les autres parties édifiées s'échelonnent entre 1789 et 1790, connaissant un rythme moins soutenu, dû essentiellement au déclenchement de la Révolution et retardant ainsi la fin des travaux ${ }^{10}$.

Le tracé du mur des Fermiers généraux, établi définitivement en 1790, ne changera guère jusqu'en 1860, à l'exception de quelques barrières qui seront nouvellement créées. La plupart des ouvrages reproduisent ainsi la carte du mur édifié à la fin du XVIII ${ }^{\mathrm{e}}$ siècle, oubliant de préciser qu'en juillet 1789 , certaines barrières au nord et dans la partie orientale de la ville ne sont pas encore construites ${ }^{11}$. Une première difficulté apparaît alors, celle de repérer précisément les barrières. Ainsi, lorsqu'on évoque les barrières Blanche, celle de Montmartre, ou encore la barrière Poissonnière, celles-ci se situent, à juste titre, au niveau actuel des boulevards de Clichy et de Rochechouart. Cependant, ceci n'est vrai qu'à partir de 1790. Il n'en est rien en 1789. À cette date, l'octroi se perçoit plus au sud, entre les rues Saint-Lazare, Coquenard et d'Enfer (c'est-à-dire entre les rues Lamartine et Bleue). L'erreur est d'autant plus facile à faire car la barrière Blanche, par exemple, porte un nom identique, aussi bien en 1789 et en 1790, alors que la limite de l'octroi n'est pas située au même endroit aux deux dates.

\section{La « prise » des Barrières en juillet 1789}

Paris est en effervescence en ce début d'été. Les incidents se font de plus en plus nombreux au cœur de la capitale dans ce Palais-Royal acquis aux patriotes et sujet aux rumeurs les plus folles. En ce début de juillet, la fermentation est vive dans la propriété du duc d'Orléans. Les limites de la ville, les barrières, continuent à avoir leur lot d'accrochages alors que des troupes du Royal-Allemand s'emploient à aider les commis de la Ferme à repousser la population qui tente de faire pénétrer dans la ville des denrées alimentaires ${ }^{12}$.

Depuis le début de l'année 1789, plusieurs signes avant-coureurs laissent percevoir une action d'envergure au nord de la capitale, où des

(10) Jean-Marc PEYSSON, op. cit.

(11) Le mot barrière est ici un terme générique ; il renvoie de manière indistincte aux bâtiments de l'octroi (locaux d'habitation, perception, hangars, ainsi qu'aux grilles d'accès). Pour ces barrières édifiées et leur mise en service à des périodes différentes, voir la carte 1 en annexe.

(12) Jacques GODECHOT, La prise de la Bastille, le 14 juillet 1789, Paris, Gallimard, 1989, p. 282-293 ; pour une relation complète des troupes qui se massent autour de Paris, voir Pierre CARON « La tentative de contre-révolution de juin-juillet 1789 », RHMC, vol. 8, nº 1, 1906-1907, p. 5-34. 
groupes d'individus, des fraudeurs, tentent de faire passer des marchandises à l'intérieur de la ville et se heurtent aux forces de l'ordre. En février, des employés de la Ferme, escortés de gardes-françaises se rendent dans un cabaret à la Haute-Courtille, où ils sont agressés à coups de pierres, lancées d'une fenêtre de l'immeuble. Les employés sortent les sabres et les fusils. Le bilan se solde par plusieurs blessés du côté des forces de l'ordre et un tué parmi les fraudeurs ${ }^{13}$. Le 4 mai, quinze personnes essaient de passer en force au nord de la capitale, voulant introduire des marchandises à l'intérieur de la ville, mais se heurtent à la brigade de Saint-Denis ${ }^{14}$. Deux semaines plus tard, des attroupements se produisent à la barrière Clichy, où la population refuse de payer les droits d'entrée ${ }^{15}$. De nombreux autres accrochages émaillent cette période, sans avoir pour autant la même intensité dramatique. Le pouvoir est inquiet de cette situation. La Ferme fait appel de plus en plus aux gardes-françaises et elle demande la création de corps de garde supplémentaires dans ces quartiers périphériques de la $v^{v i l l e}{ }^{16}$. Dans ce climat, où tout est possible, c'est une occasion extrêmement favorable pour certains Parisiens d'agir. Le renvoi de Necker, connu par la population le 12 juillet, précipite les événements connus.

Il me paraît important d'examiner le déroulement de ces journées de juillet et de rendre compte de cette dynamique qui rend possible les événements à venir. Plus qu'une émeute sans lendemain, la population, faute d'être entendue, s'exprime ouvertement, violemment. Mais il serait trop simple et illusoire d'imputer exclusivement l'attaque des barrières à des brigands, à des « fraudeurs professionnels $»^{17}$.

Il n'est pas possible dans le cadre de cet article d'examiner tous les incidents qui eurent lieu durant ces journées de juillet $1789^{18}$. Les cartes (en annexe) permettent de rendre compte de l'intensité des faits, de

(13) $\mathrm{AN}, \mathrm{Z}^{1 \mathrm{G}} 217$, information à la requête de Mager, adjudicataire des Fermes du Roy contre Cœur de Bois l'ainé et autres. Cette sous-série concerne les diverses informations judiciaires traitées par les cours de l'Election et des Aides durant l'année 1789.

(14) Ibidem, Y 18795, les individus arrêtés sont écroués à la Force.

(15) Ibid., $\mathrm{O}^{1}$ 500, incidents du 19 au 22 mai 1789.

(16) Ibid., $\mathrm{O}^{1} 500$, f. 81,2 février 1789, création d'un corps de garde au niveau de la barrière Monceau ; Ibid., f. 132, 162, 179, 20 février-2 mars 1789, augmentation des effectifs des gardes-françaises aux barrières de Monceau et Clichy.

(17) Cette appellation, utilisée par la Ferme, désigne ceux qui en font leur activité principale.

(18) Je n'examinerai pas davantage le rôle des gardes-françaises qui n'ont exercé aucune répression contre les émeutiers aux barrières mais se sont cantonnés à montrer leur présence, jouant la fonction de "tampon », en évitant des incidents trop violents. Toutefois, on ne peut parler de fraternisation comme ce fut le cas lors de la prise de la Bastille. De même l'identité des acteurs aux barrières n'est pas abordée, seulement suggérée. Le rôle de la cour des Aides, juridiction financière qui juge les affaires relatives aux impôts et à la fraude, ne sera pas davantage traité ; enfin, la procédure judiciaire engagée par la cour des Aides contre les incendiaires est seulement effleurée. 
mesurer quelles sont les barrières touchées et à quel moment elles le furent. Cependant, on peut esquisser une chronologie de l'attaque aux barrières en privilégiant le nord de la capitale, là où tout a commencé.

Le premier accroc ne fait pas, à proprement parler, partie de l'attaque contre les barrières, car il n'y a pas une action directe contre les bureaux. Néanmoins, il inaugure et marque le déclenchement des nombreux incidents qui se déroulent pendant plusieurs jours et nuits, et dont l'acte final se clôt avec la prise de la forteresse au faubourg Saint-Antoine. Dans la nuit du 9 au 10 juillet, un accrochage se produit à proximité immédiate de la barrière Montmartre. Une inspection menée dans une maison par les employés de la Ferme tourne à la déroute pour les commis, car plusieurs centaines d'ouvriers, embauchés aux ateliers de charité s'ameutent et les commis sont obligés de fuir ${ }^{19}$. Mais c'est véritablement le lendemain que l'épisode des barrières prend une ampleur tumultueuse.

Les premiers bureaux touchés sont attaqués au nord, au nord-est et à l'est de Paris, dans la nuit du 10 juillet aux endroits où le mur en moellon n'est pas encore édifié. À la place de la pierre, c'est une palissade en planches de bois qui fait office de séparation entre la banlieue et Paris et marque ainsi la limite fiscale. Les employés dans cette zone sont logés dans des maisons louées, situées aux environs immédiats des barrières et le personnel veille à la perception dans des guérites en bois qui peuvent être transportées aisément. Le vendredi 10 juillet, vers 21 heures, à la barrière Papillon, des pierres, lancées d'un immeuble, s'abattirent sur les employés alors que ceux-ci fouillaient un individu soupçonné de transporter des vessies d'eau-de-vie. Les commis s'abritèrent précipitamment dans leur bureau ${ }^{20}$. Le même soir vers 23 heures, à la barrière Saint-Georges et des Trois-Frères, les employés placèrent deux roulettes d'observation (des guérites) rue Saint-Lazare, près de la rue La Rochefoucauld, ayant constaté depuis plusieurs nuits que des marchandises étaient introduites en fraude dans la ville. Une dizaine d'individus s'approcha d'une des roulettes ; ils s'adressèrent calmement au sous-brigadier, lui indiquant que, dans moins de 24 heures, elles n'y seraient plus. Parole tenue car, dès le lendemain, la barrière et les roulettes furent incendiées ${ }^{21}$. Le lendemain,

(19) $\mathrm{AN}, \mathrm{Z}^{1 \mathrm{G}} 217$, procédure à l'Élection de Paris, information du 14 août 1789.

(20) Ibidem, $\mathrm{Z}^{1 \mathrm{~A}} 886$, Information du 29 mars 1790 et jours suivants concernant l'incendie des barrières, $30^{\text {ème }}$ témoin, à la barrière Papillon ; ce bureau se trouvait au niveau de l'actuelle rue La Fayette, en face du square Montholon et, de chaque côté de la voie, la clôture en bois marquait la séparation entre le taillable et la zone sujette aux droits.

(21) Ibid., Information.., $28^{\text {ème }}$ témoin, à la barrière Saint-Georges et des Trois-Frères. 
samedi 11 juillet, à 10 heures, la barrière Saint-Martin connut les premiers véritables soubresauts. Un scénario analogue à ce qui s'était produit la veille à la barrière Papillon ébranla les employés dont le bureau échappa de peu à un incendie. À l'annonce de l'arrestation d'un homme qui transportait des vessies d'eau-de-vie, les employés virent s'abattre sur leur bureau une troupe de plusieurs centaines d'individus, armés de pioches, de pelles, de grosses pierres et de pavés. Les premiers «vive le Tiers-État» se font entendre, relayés par des avertissements à l'encontre des employés que la population menaçait de brûler. Des hommes et des femmes pénétrèrent dans le bureau, vidèrent les armoires, foulant aux pieds les effets des commis, brisèrent les vitres et commencèrent à casser les cloisons à coups de massues. Ils envisageaient de mettre le feu au bureau mais un détachement de gardes-françaises arriva sur les lieux, ce qui dissuada les émeutiers de continuer leur besogne $\mathrm{e}^{22}$.

C'est durant la nuit du 11 juillet que les assauts aux barrières sont marqués par les premiers incendies. Le premier à brûler fut le bureau de recette de la barrière Blanche, situé à l'angle de la rue Saint-Lazare et de la rue de la Chaussée d'Antin. Vers 21 heures, un grand nombre de «particuliers des deux sexes » se présenta à la barrière. Beaucoup de ces individus, parmi lesquels plusieurs femmes, tenaient et brandissaient des pinces, des merlins, des marteaux. La foule apportait toutes sortes d'objets hétéroclites en bois qu'elle disposait près du mur du bureau. Des « brigands » avaient démonté la clôture en bois qui fut mise dans le brasier disposé à quelques mètres du bâtiment. Le bureau fut ensuite investi à son tour. Le saccage à l'intérieur commença : la porte d'entrée fut éventrée, les deux tables, l'armoire, les chaises et le poêle en faïence furent jetés à l'extérieur. Les émeutiers se saisirent également des quelques registres de perception qu'ils balancèrent dans le feu. Les flammes crépitaient. Les menaces fusaient sur les employés à qui on assurait de « leur faire un très mauvais parti ». Ces intimidations furent prises au sérieux et les commis quittèrent les environs de la barrière pour aller se réfugier un peu plus loin, rue Saint-Lazare. Ce n'est que vers minuit, que deux cents gardesfrançaises arrivèrent sur les lieux. Ne demandant pas leur reste, évitant un affrontement direct, les incendiaires se replièrent aussitôt vers la barrière

(22) Ibid., procès-verbal des commis de la barrière Saint-Martin, 11 juillet 1789 ; Information..., $37^{\text {ème }}$ témoin. 
Montmartre. Mais la troupe ne resta pas longtemps, appelée ailleurs, et elle repartit vers deux heures du matin ${ }^{23}$.

Aux barrières Saint-Georges et des trois-Frères, vers 23 heures, ce 11 juillet, les commis virent arriver des «séditieux inconnus». Ils savaient ce qui se passait chez leurs collègues de la barrière Blanche. Les commis, là non plus, ne demandèrent pas leur reste et décampèrent avant l'arrivée des émeutiers, laissant l'accès libre au peuple qui franchissait sans contrôle les barrières, chargé de vessies d'eau-de-vie. Une section de gardesfrançaises survint dans le secteur. Leur seule présence empêcha là-aussi tout débordement. Les militaires patrouillèrent dans la rue, puis, moins de deux heures plus tard, évacuèrent les parages. Arriva alors une soixantaine d'individus qui fit un "vacarme épouvantable », frappant à coups de bâton et de barre de fer sur les édifices. S' adressant aux commis qui étaient revenus, ils sollicitèrent du café, de l'eau-de-vie, et leur demandèrent « s'ils étaient du Tiers-État ». En partant, malgré la réponse positive des employés, les individus dévastèrent sur leur passage la roulette en bois, installée comme poste d'observation. Le lendemain matin, face à de nouvelles incursions, les employés de la Ferme firent appel aux gardes-françaises de la caserne Poissonnière qui vinrent appuyer ceux du faubourg Saint-Martin ${ }^{24}$.

À la barrière de Clichy, le 12, en milieu d'après-midi, les commis assistent aux mêmes scènes qui voient les barrières saccagées par une masse importante d'individus toujours armés d'instruments contondants et tranchants ${ }^{25}$. Ce sont ainsi les barrières de la partie septentrionale qui subissent les premiers assauts.

Des scènes identiques se répètent les jours suivants aux quatre points cardinaux de la capitale. Les barrières sont pillées, saccagées, détruites. Quelquefois, un deuxième assaut s'avère nécessaire où après avoir, dans un premier temps, dévasté les bâtiments, ces derniers sont ensuite incendiés quelques heures plus tard ou le lendemain, lorsqu'il n'y a plus de menaces d'intervention des troupes. Ainsi, à l'aube du dimanche 12 juillet, les individus qui avaient assailli la veille la barrière Blanche, retournaient au bureau de recettes et se ruèrent à l'intérieur du bâtiment qu'ils vidèrent

(23) Ibid., procès-verbal des commis de la barrière Blanche ; Information..., $1^{\mathrm{er}}, 2^{\text {ème }}, 3^{\text {ème }}$, $4^{\text {ème }}$ et $5^{\text {ème }}$ témoin ; les témoignages sont légèrement divergents sur l'heure exacte de l'attaque contre la barrière Blanche ; certains parlent de $20 \mathrm{~h}$, d'autres font débuter l'assaut à $21 \mathrm{~h}$.

(24) Ibid., procès-verbal de séditieux inconnus, pour voies de faits, du 11 juillet 1789, à la barrière Saint-Georges.

(25) Ibid., Information...4ème, 5ème et 6ème témoin. 
cette fois-ci entièrement, à l'exception des matelas qui furent rendus aux employés de la Ferme ${ }^{26}$.

La barrière de Fontainebleau, à l'emplacement sud de l'actuelle place d'Italie, subit des dégradations importantes. Le 12 juillet au soir, le receveur des entrées se rend sur place, voulant vérifier par lui-même si les bureaux avaient subi des dégâts. Il n'en était rien. Les familles logeant dans les bâtiments n'étaient cependant pas rassurées. Le receveur comptait bien sur la maréchaussée de Villejuif pour parer à toute éventualité. Dans la nuit, il se risqua au dehors du côté du fleuve, mais la nuit paraissait calme. Le lendemain, au petit matin, une voix de femme le réveilla en sursaut, l'informant que des hommes se dirigeaient vers le bâtiment. « Nous sommes tous perdus », ajouta-t-elle. Sortant sur le perron, le receveur vit une « troupe de brigands ayant l'air très déterminé ». Des individus, munis de quelques armes à feu, avaient réussi à pénétrer dans le bâtiment en défonçant la porte et s'emparèrent des fusils de service des employés. Toute résistance devenait inutile, voire périlleuse. Les cloisons de séparation furent démolies à coups de merlins et de haches, puis le feu fut mis à l'escalier central ${ }^{27}$.

Une importante foule, venant aussi bien de l'extérieur que de l'intérieur de la ville, s'empara des divers bâtiments ce 13 juillet. Les employés peuvent à ce moment distinguer les flammes qui s'élèvent dans le ciel, provenant des bureaux limitrophes, situés à quelques centaines de mètres : à l'ouest, la barrière du Clos-Payen (Croulebarbe) ; au nord-ouest, celles de l'Hôpital (des deux Moulins) et d'Ivry et enfin le bureau de la Gare au bord de la Seine. La perception des droits ne se faisait pas uniquement sur le pourtour de la ville : d'autres endroits, sur le fleuve, servaient également de recouvrement des taxes des marchandises. Il existe ainsi, en amont et en aval du fleuve, des embarcations (les pataches) qui contrôlent les navires mouillant dans les ports de la ville. Les pataches du quai de la Râpée furent incendiées ce 13 juillet ${ }^{28}$.

S'agit-il des mêmes groupes qui se portent à plusieurs barrières ? Ces dernières sont relativement proches les unes des autres, éloignées de quelques centaines de mètres pour les barrières méridionales et, pour celles de la partie septentrionale, les distances sont encore plus courtes. Les mouvements de foule sont aisément perceptibles et le feu qui se dégage se voit d'assez loin. Les attroupements qui se portent aux barrières nord de la

(26) Ibid., procès-verbal des commis de la barrière Blanche.

(27) Ibid., procès-verbal des employés à la barrière de Fontainebleau, 13 juillet 1789 ; Information..., $45^{\text {ème }}, 58^{\text {ème }}, 59^{\text {ème }}$ témoin.

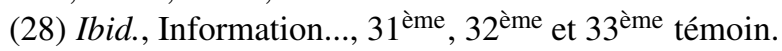


capitale sont le fait très souvent de mêmes groupes d'individus. Des bandes que l'on voit à telle barrière se retrouvent à une autre : quelques minutes plus tard. Entre le moment où une barrière est saccagée, voire brûlée, et une autre, peu de temps s'écoule, une heure, deux heures au plus. C'est le cas entre les barrières du Roule et de Sainte-Anne (actuel angle de la rue La Fayette et de la rue du faubourg-Poissonnière). Les quartiers dans la partie orientale - à Belleville notamment - sont touchés d'une façon similaire : des groupes composés de mêmes individus se déplacent le long de la clôture en bois et remontent vers le nord. Au sud, les employés de la barrière Fontainebleau distinguaient clairement plusieurs groupes qui quittaient le bureau de la Gare. L'un se dirigeait vers eux directement, pendant qu'une autre formation faisait halte dans le bureau limitrophe.

Les fraudeurs, tout au long de ces journées, anticipent les événements, jaugent les employés. Calmement, ils s'adressent aux commis, leur conseillent de filer, indiquent que les barrières vont être incendiées, que des marchandises vont être introduites dans la ville. À la barrière Clichy, le 13 juillet, en début d'après-midi, quelques hommes venant du côté du bureau Blanche s'arrêtèrent devant le bâtiment et avertirent les employés qu'une voiture remplie de vin allait passer. Effectivement, moins d'une heure plus tard, l'attelage entrait sans rencontrer d'obstacle, escorté de plusieurs hommes armés de gros bâtons ${ }^{29}$. Dans la partie méridionale, le 13 juillet au soir, à la barrière Vaugirard, un homme faisait rouler un tonneau, suivi de plusieurs autres personnes. Les employés esquissent un geste, montrant leur intention de le contrôler, mais peine perdue devant la réplique cinglante des individus : « vous n'avez que faire ici, nous allons revenir ». Effectivement, un peu avant minuit, les commis reconnaissaient certains individus qui roulaient plusieurs tonneaux ${ }^{30}$.

Le mode opératoire est donc sensiblement similaire à toutes les barrières de l'octroi. Un premier temps, un passage initial où les différents groupes se portent aux barrières, en montrant aux employés leur détermination, étalant leur force et leur supériorité numérique. Cela suffit dans la plupart des cas pour que les employés déguerpissent. Prévenir les commis que l'on va revenir plus nombreux ou que l'on va faire passer des marchandises permet d'apprécier si la riposte adverse sera à la mesure. Devant la reculade des employés, les fraudeurs comprennent vite qu'ils ont

(29) Ibid., Information..., $4^{\text {ème }}, 5^{\text {ème }}$ témoin. L'un des chefs fraudeurs, Lefèvre, dit Cœur de bois, dirige les opérations ; il sera considéré comme un des principaux responsables par la Cour des Aides en 1790.

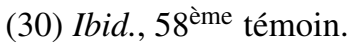


le champ libre. Ils peuvent ainsi revenir le soir même ou le lendemain et mettre à exécution leur dessein : mettre le feu aux meubles et bureaux.

\section{La prise des barrières : les fraudeurs et le peuple de Paris unis dans un même combat}

Affaire de fraudeurs, certes. Mais la population des quartiers n'est pas absente de ces événements. Ici, il faut être plus nuancé. Si le peuple ne participe pas à l'assaut directement, du moins, il ne réagit pas contre les dégradations. Ce qui se déroule sous les yeux des Parisiens laisse apparaître plutôt une acceptation. À la barrière du Roule, pendant que le saccage avait commencé à l'intérieur du bureau, une partie de « la population et les voisins disaient qu'il fallait assassiner les commis $»^{31}$. Aux barrières du Temple et de la Courtille, c'est un cri unanime qu'entendent les employés « nous ne voulons plus de barrières ni de commis ». Des dizaines de badauds se sont assemblés, acclamant et observant ce qui se déroulait sous leurs yeux ${ }^{32}$. Alors que des hommes et des femmes défilent, brandissant une effigie à la barrière Blanche, des habitants du quartier se joignent au groupe qui saccage le bureau ; ils applaudissent, crient, expriment leur contentement de la scène vécue $^{33}$. Après la prise de la Bastille, le 15 juillet, les employés étaient tout surpris de constater que « la population et les fraudeurs montaient la garde ensemble en disant clairement qu'ils ne voulaient plus de commis $»^{34}$. C'est plutôt une connivence qui s'installe entre la population, les fraudeurs et la garde bourgeoise : à la barrière du Marais, alors que la future garde nationale patrouille en vérifiant si des armes pénètrent dans la ville, les marchandises introduites circulent librement ${ }^{35}$. Au demeurant, il est peu probable que la population n'ait pas réagi. L'effet d'entraînement a dû jouer en grande partie. L'indécision a certainement peu duré et il est aisé de supposer que les Parisiens ont compris bien vite l'intérêt qu'il y avait à détruire les barrières qui représentaient un obstacle majeur, obligeant la population à acquitter des droits et les empêchant de se mouvoir librement.

Le mot « foule » revient abondamment dans les témoignages mais le terme, trop vague, est utilisé sans que l'on sache toujours quels groupes sociaux la composent. Le peuple parisien parle-t-il d'une seule et même voix, faisant cause commune avec les fraudeurs ? Ce dernier mot renvoie

(31) Ibid., $18^{\text {ème }}$ témoin.

(32) Ibid., $40^{\text {ème }}, 41^{\text {ème }}$ témoin.

(33) Ibid., $27^{\text {ème }}, 28^{\text {ème }}$ témoin.

(34) Ibid., $41^{\text {ème }}, 42^{\text {ème }}$ témoin, à la barrière du Temple.

(35) Ibid., procès-verbal des employés de la barrière du Marais, le 12 juillet 1789. 
à une classification particulière, désignant ceux qui sont en rapport direct avec le monde du vin et de l'eau-de-vie, vivant directement de ce commerce illicite. Ce sont tous ceux que les procès-verbaux désignent par l'expression de « fraudeurs de profession ». Évoquer le peuple dans son ensemble, c'est vouloir s'affranchir d'une définition précise qui renvoie à des catégories complexes d'individus. On ne peut donc en faire l'économie. Je retiendrai ici la formulation la plus couramment admise qui suggère le monde du travail salarié, à commencer par les plus humbles d'entre eux, les gagnedeniers et journaliers, qui peuvent basculer dans la marginalité rapidement, sans oublier les ouvriers ou les compagnons dont le sort n'est pas toujours enviable. La liste n'est pas exhaustive mais la procédure engagée par la Cour des Aides en 1790 contre quatre-vingts individus décrétés de prise de corps reste lacunaire et toutes les personnes accusées ne purent être identifiées. Il faut ajouter à ces individus soupçonnés d'avoir participé aux incendies des barrières, quinze personnes qui furent assignées pour être entendues par la Cour des Aides. L'identité est connue pour soixante personnes. Dix individus font partie de ce groupe de fraudeurs, quinze autres exercent le métier de marchands de vins ou sont assimilés comme tels. La grande masse des individus baigne dans ce monde de déchargeurs, porteurs d'eau, ouvriers des ports ou des ateliers de charité, entourés d'un nombre moindre de boutiquiers et artisans ${ }^{36}$. Quelques déclarations indiquent que certains émeutiers ne faisaient pas partie du menu peuple, et que l'on peut les classer comme étant des bourgeois. Les employés de la Ferme évoquent des hommes «assez bien vêtus », portant « une redingote blanche » ou tenant « une canne à pomme d'or $»^{37}$.

Une rapide mise en perspective permet de percevoir que, socialement, ces individus ne différent guère de ceux que l'on rencontre lors du sac du couvent Saint-Lazare ou au moment de la prise de la Bastille ${ }^{38}$. La seule véritable différence (mis à part les gardes-françaises) est constituée par le groupe de marchands de vin qui se trouve surreprésenté. À y regarder de près, ce phénomène n'est pas surprenant. Les cabarets et autres guinguettes

(36) Ibid., procédure contre 80 individus soupçonnés d'avoir participé aux incendies des barrières en juillet 1789, arrêté de la Cour des Aides, 10 mai 1790.

(37) Ibid., Information..., $15^{\text {ème }}, 17^{\text {ème }}, 27^{\text {ème }}$ témoin. Parmi ces individus à l'allure aristocratique, un personnage haut en couleur se distingue nettement : Musquinet de Saint-Félix ; celui-ci, originaire de Pontoise, est sous les verrous depuis le printemps 1790 à la Conciergerie pour avoir incité les paysans de l'Oise à se révolter contre les impôts. Son procès sera finalement joint à celui des incendiaires de juillet 1789, car Musquinet est soupçonné d'avoir joué un rôle moteur durant ces journées aux barrières sud de la ville.

(38) Pour une analyse détaillée de la composition des foules, voir Georges RUDÉ, op. cit. et « La composition sociale des insurrections parisiennes de 1789 à 1791 », AHRF, 24, 1952, p. 256-288. 
jouxtent le mur des Fermiers du côté extérieur, vers la banlieue. Ce sont ainsi des dizaines de débits de boissons qui sont situés aux portes de Paris accueillant les Parisiens ${ }^{39}$. Les marchands de vin et cabaretiers ont suivi l'évolution de la construction de la clôture et ont saisi depuis longtemps que l'espace dans lequel ils vivaient et exerçaient leur activité se retrouverait dans la ville intra-muros. La franchise des droits dont ils profitaient allait donc s'éteindre. Parmi les quatre-vingts individus, une trentaine réside dans les faubourgs Montmartre, Poissonnière, Saint-Laurent ou à la Courtille. L'intérêt de voir disparaître le mur des Fermiers et avec lui les taxes est indéniable.

Cependant, si la composition sociale semble similaire, cela ne signifie pas que les émeutiers aux barrières sont présents sur d'autres lieux dans la capitale. Faute de données tangibles, il est malaisé de se prononcer sur cette question, quoique Haim Burstin rappelle les propos que tenait Restif de la Bretonne à la vue de ces groupes se dirigeant vers l'enclos de Saint-Lazare et qui venaient des barrières de l'est parisien ${ }^{40}$. Dans le même ordre d'idées, la Cour des Aides avait lié ces deux attaques (l'incendie des barrières et le pillage de la maison Saint-Lazare) dans une procédure unique ${ }^{41}$. Pour la juridiction, il semble que le doute n'était pas permis et que de nombreux émeutiers avaient sévi simultanément dans ces deux périmètres.

\section{La revanche du peuple : s'inscrire durablement dans l'espace public}

De nombreux Parisiens goûtent à l'humidité et à l'obscurité de la fameuse prison parisienne sur l'île de la Cité, lorsqu'ils sont conduits à la Conciergerie pour fraude sur le vin ou l'eau-de-vie ${ }^{42}$. Ce qui n'empêche pas certains de continuer leurs affaires après leur libération, quitte à se retrouver de nouveau derrière les barreaux. Vivre de la fraude est un moyen comme un autre, du moment qu'il permet de glaner quelques pièces, de nourrir sa famille, sans pour autant se considérer comme un criminel. À un moment où la population attendait beaucoup de la réunion

(39) Pour la sociabilité populaire dans les cabarets, Thomas BRENNAN, Public Drinking and Popular Culture in Eighteenth-Century Paris, Princeton University Press, 1988 ; pour « la culture de l'enivrement » et les oppositions à l'ivresse, voir Matthieu LECOUTRE, Ivresse et ivrognerie dans la France moderne, Rennes, PUR, 2011.

(40) Haim BURSTIN, op. cit, p. 58.

(41) AN, $Z^{1 \mathrm{~A}} 886$, procédure de la Cour des Aides qui sursoit à l'instruction des incendies des barrières de juillet 1789 et du couvent Saint-Lazare, 30 juin 1790.

(42) Les registres des écrous de la Conciergerie demeurent à cet égard éloquents ; on ne peut que constater la place énorme qu'occupe la fraude dans le paysage urbain de la capitale entre 1785 et 1791, APP (Archives de la Préfecture de Police), AB 128 à 132 ; en 1789, par exemple, c'est en moyenne une personne par jour qui est écrouée pour fraude à la Conciergerie. 
des États généraux, où les ordres ont été convoqués pour tenter de résoudre justement la crise financière, c'est un visage bien sombre que dévoile la Ferme. Las de ces sacrifices incessants, la population attend le moment favorable pour s'exprimer, en entrant dans l'espace public ${ }^{43}$. Ceci se manifeste ouvertement aux barrières. Cela ne signifie pas forcément que cette population porte un projet politique cohérent, quoique certains aient signifié à la Ferme, l'iniquité de ces taxes ${ }^{44}$. Les attaques aux barrières ne sont pas exclusivement, quoi qu'en dise la Cour des Aides, le fait de bandits ou de brigands. Quand bien même ces derniers seraient présents en grand nombre aux portes de Paris, cela change-t-il objectivement le sentiment d'injustice vécue par la population depuis la construction du mur ? L'arme principale demeure cette cohésion du groupe qui lutte, utilisant au besoin la violence qui n'est pas une fin en soi, mais un moyen pour parvenir au but recherché. Au demeurant, la force n'a pas besoin d'être exposée continuellement, elle constitue déjà une menace et cela suffit dans la plupart des cas pour que la démonstration de cette puissance ne soit pas étalée au grand jour ${ }^{45}$. Indéniablement, face à une conjoncture explosive, une partie de la population a su tirer parti des événements, afin d'être en mesure de réussir cette formidable gageure, mettre à bas la Ferme et sa volonté d'enfermer les Parisiens.

L'ensemble des faits ouvre des perspectives inédites jusque-là. Nous sommes loin de ces explosions irrationnelles qui semblent caractériser les foules. Les actions menées peuvent être compréhensibles car elles ont un sens et prennent une envergure politique. La foule n'est plus cet agrégat d'individus, pas plus qu'un attroupement plus ou moins spontané, mais elle est le résultat d'un rassemblement volontaire, car ceux qui s'unissent participent d'une volonté et d'un but qui vont dans le même sens ${ }^{46}$.

(43) Pour cette notion, voir Jurgen HABERMAS, L'espace public, archéologie de la publicité comme dimension constitutive de la société bourgeoise, trad. fr., Paris, Payot, réed. 1993 ; Keith Michael BAKER, Au tribunal de l'opinion : essais sur l'imaginaire politique au XVIII ${ }^{e}$, trad. fr., Paris, Payot, 1993 ; Raymonde MonNIER, L'espace public démocratique : essai sur l'opinion à Paris de la Révolution au Directoire, Paris, Kimé, 1994.

(44) Étienne Chevalier, député aux États généraux, vigneron dans la vallée de Montmorency, a rédigé de nombreux mémoires et ouvrages dans lesquels il dénonce le poids des taxes. Il sera un des chefs de file à l'Assemblée nationale pour demander la suppression des droits d'octroi. De même, les marchands de vin du nord et de l'est de la capitale s'étaient regroupés au début de l'année 1789, signant un acte devant notaire, et affirmant leur refus de voir leurs quartiers intégrés au mur des Fermiers, car les cabarets se retrouvaient de fait assujettis aux taxes. Plusieurs cahiers de doléances du Tiers de la ville et de la banlieue se prononcent contre les taxes aux barrières.

(45) Richard COBB, La protestation populaire en France, 1789-1820, trad.fr., Paris, CalmannLévy, 1975, deuxième partie essentiellement.

(46) Georges LefEBVRE, Études sur la Révolution française, Paris, P.U.F, 1963, p. 371-392 (il s'agit de l'article sur les foules révolutionnaires, publié antérieurement dans les $A H R F$ ). 
La violence de ces individus est réelle, mais contrôlée. À maints endroits, les commis, s'ils sont inquiétés, insultés ou menacés de " griller », ne sont cependant pas assommés ou violentés. À la barrière de Montreuil, les incendiaires leur conseillent de partir avant que le feu ne soit allumé ${ }^{47}$. Le lundi 13 juillet, à la barrière de Fontainebleau, les employés demandent aux émeutiers de quitter les lieux, emportant avec eux leurs effets personnels, ce qui leur est accordé ${ }^{48}$. Lorsque le 14 juillet, un individu prend au collet un commis à la barrière de Chaillot et semble menaçant avec un sabre à la main, il lui demande avec une pointe d'humour s'il veut continuer à travailler à ses risques et périls ou s'il préfère partir sain et sauf, l'employé alors ne demande pas son reste et l'homme le laisse détaler tranquillement ${ }^{49}$. Aux questions récurrentes et provocantes des séditieux, il suffit que les employés des barrières répondent qu'ils sont du tiers état, pour déguerpir sereinement.

Les flammes qui montent dans le ciel de Paris sont impressionnantes. Le danger est imminent ; il suffit que le vent souffle brutalement et le feu risque de se propager à la ville. Là encore, les incendiaires font preuve de retenue et ne prennent pas le risque de voir le feu s'étendre. Aux barrières nord de la capitale, la clôture en bois, jetée au feu, est toute proche des immeubles, elle en fait la jonction. Le risque d'une propagation de l'incendie aux bâtiments d'habitation est trop grand pour que les habitants ne réagissent pas. Sur la demande de plusieurs particuliers, les incendiaires enlevèrent les lattes de bois ${ }^{50}$. Pendant que la barrière de la Voirie se consumait, les gardes-françaises obtiennent de ces « malheureux » qu'ils brûlassent les effets un peu plus loin à cause de la proximité d'autres habitations qui pouvaient à tout moment prendre feu. C'est un dialogue indirect qui s'installe entre les émeutiers et la troupe mais aucunement une volonté répressive de la part des militaires. Ce fut une des priorités des gardes, celle d'éviter la propagation de l'incendie ${ }^{51}$.

Un véritable cri de ralliement lie la population qui se porte aux barrières. Le vent de liberté souffle et les exhortations se font entendre. «Êtes-vous du Tiers-État? » clament haut et fort les émeutiers aux commis de la barrière Saint-Georges dans la nuit du 11 au 12 juillet ${ }^{52}$. Les employés juillet 1789 .

(47) $\mathrm{AN}, \mathrm{Z}^{1 \mathrm{~A}} 886$, procès-verbal des employés de la Ferme à la barrière de Montreuil, 13

(48) Ibid., $65^{\text {ème }}$ témoin.

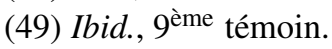

(50) Ibid., 30 ème témoin.

(51) Ibid., procès-verbal des commis de la barrière de la Voirie, le 12 juillet 1789.

(52) Ibid., Information..., $27^{\text {ème }}, 28^{\text {ème }}, 29^{\text {ème }}$ témoin, à la barrière Papillon. 
entendent venir de loin cette foule qui gronde sa colère en frappant les pavés parisiens de ses grosses massues et menaçant les employés ouvertement : « où sont-ils [les commis] ? Il faut les tuer », «c'est le Tiers-État qui arrive » tonne fortement la population ${ }^{53}$. Paroles qui deviennent obsédantes et semblent justifier leur entreprise de vouloir tout saccager au nom d'un droit oublié, d'un droit naturel, celui de garantir une vie décente. Une nouvelle validation prend naissance à partir de ces actions qui s'élaborent et où le troisième ordre saisit une place qu'il n'avait pas jusque-là. La demande, voire l'exigence est claire. Un poids fiscal qui grève de plus en plus le budget de la population, une liberté de déplacement entravée, des fouilles en règle, peut-être même trop appuyées lorsqu'il s'agit d'une femme, bref trop d'humiliation qui rend insupportable ce mur qui bouche l'horizon. Il est donc temps d'ouvrir enfin ces barrières, se frayer un passage ininterrompu, pour « goûter à la liberté $»^{54}$.

Quand la foule, indifféremment mêlée d'hommes et de femmes, construit un mannequin à l'effigie d'un brigadier des Fermes, le promène près de la barrière et le brûle, elle montre son hostilité à l'égard d'un homme plus particulièrement, mais marque autant son exécration face à des impôts injustes. Les femmes, ici, jouent un rôle de premier plan. L'une d'elles crie distinctement son animosité envers cet employé, clamant sa volonté de le brûler. Elle porte, avec d'autres, ce pantin en paille ${ }^{55}$. Tout comme en avril 1789, lorsqu' un homme brandissait un écriteau qui reprenait une formule similaire pendant que la foule promenait deux mannequins représentant les deux entrepreneurs, Réveillon et Henriot ${ }^{56}$. La Picarde (tel est son surnom) n'est pas en reste et dirige une troupe d'une vingtaine de personnes, mettant le feu aux bureaux et emportant plusieurs centaines de bouteilles de vin entreposées dans la cave ${ }^{57}$. La fête est présente. On célèbre ce que l'on croit être la fin d'un monde inégalitaire. La musique fait partie également de ces réjouissances au son des tambours et violons qui accompagnent la destruction des bâtiments et des barrières ${ }^{58}$. Ces

(53) Ibid., procès-verbal des employés de la Ferme à la barrière de Reuilly, le 13 juillet 1789 ; Information..., $40^{\text {ème }}, 41^{\text {ème }}$ témoin, barrière du Temple, le 12 juillet $1789 ; 42^{\text {ème }}$ témoin, barrière de Montmartre, le 12 juillet 1789.

(54) Ibid., Information..., 45 ème témoin, barrière du Clos-Payen, le 13 juillet 1789.

(55) Ibid., procès-verbal des commis de la barrière Blanche ; la femme en question crie «brûlons Demilhac [le brigadier] ».

(56) Jacques GODECHOT, op. cit., p. 224.

(57) AN, $\mathrm{Z}^{1 \mathrm{~A}} 886$, Information..., $12^{\mathrm{ème}}$ témoin ; cette femme, cardeuse de matelas, est arrêtée en juin 1790, pour sa participation aux incidents.

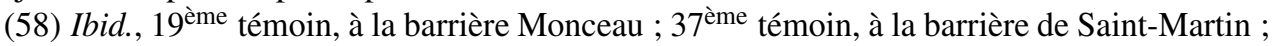
procès-verbal des employés à la barrière de Sèvres, le 12 juillet 1789. 
processions font appel à la mémoire collective du charivari certes, mais s'orientent vers un autre objectif où la population ameutée donne du sens aux événements qu'elle a décidés ${ }^{59}$.

L'émeute pouvait apparaître comme une forme classique de la protestation populaire. Mais sa répétition simultanée à la quasi-totalité des barrières ouvre d'autres perspectives d'analyse : loin d'être une simple contestation, ces incidents s'inscrivent dans une prise de conscience politique $^{60}$. Il y a chez ces hommes et ces femmes, une organisation certaine, un répertoire d'action avec certains moyens pour réaliser ce qu'ils souhaitent, pour reprendre la notion développée par Charles Tilly ${ }^{61}$. La foule, de façon plus ou moins spontanée, montre les diverses formes de cette action : en passant par les cris, le fracas des massues et autres objets sur les façades des bâtiments, les insultes, l'intimidation, le saccage et le pillage, et en dernier recours, l'utilisation du feu. Moment hautement purificateur car le mur des Fermiers et les pavillons de l'octroi laissent leur empreinte, non en un seul secteur mais sur la totalité de l'espace. Poussée par le besoin de se nourrir certes, cette population « d'en bas » impose aussi son histoire, celle de jouer un rôle dans le déroulement des événements, de participer à une nouvelle histoire qui se construit. Ce stimulus économique, le manque de pain, ferait réagir les individus sans qu'ils aient une pensée construite, sans objectifs conscients ${ }^{62}$. C'est ce que dit implicitement Clément de Barville, le procureur général de la Cour des Aides, lorsque la procédure contre les incendiaires de juillet 1789 débute à la fin de l'hiver 1790. Selon lui, mis à part les « principaux coupables » qui sont des fraudeurs notoires, ceux qui ont participé à l'incendie des barrières «n'ont été entraînés que

(59) Sur le charivari, la révolte et le lien avec la violence, voir Yves-Marie BERCÉ, Fête et révolte, Des mentalités populaires du XVIe au XVIII ${ }^{e}$ siècle, Paris, Hachette littératures, 1976 ; Le charivari, Actes de la table ronde organisée à Paris (25-27 avril 1977) publiés par Jacques LE GoFF et Jean-Claude SchmitT, Paris, La Haye, Mouton, 1982 ; William BEIK, « The violence of the french crowd from charivari to Revolution », Past and Present, vol. 197-1, 2007, p. 75-110.

(60) Jean NiCOLAS, op. cit., p. 27. Je n'examinerai pas ici les notions développées par l'auteur qui sont au centre de son analyse, à la recherche de ces bruits, murmures, émotions, émeutes, rébellions, révoltes.

(61) Charles TILly, La France conteste de 1600 à nos jours, trad.fr, Paris, Fayard, 1986.

(62) Je n'entrerai pas ici dans l'analyse de ce que veut la foule et comment elle peut l'obtenir, ce qui renvoie à la notion de l'économie morale, initiée par Edward Palmer THOMPSON, et reprise depuis par de nombreux historiens et sociologues ; Edward Palmer THOMPSON, «The Moral Economy of the English crowd in Eighteenth Century », Past and Present, vol. 50, 1971, p. 76-136 ; voir la trad. fr, «L'économie morale de la foule dans l'Angleterre du XVIII ${ }^{\mathrm{e}}$ siècle » dans Edward Palmer THOMPSON, Florence GAUTHIER, Guy-Robert IKNI et alii (éd.), La guerre du blé au XVIII siècle. La critique populaire contre le libéralisme économique au XVIII ${ }^{e}$, Montreuil, Éditions de la Passion, 1988, p. 31-92. 
par une ivresse involontaire et un délire passager $»^{63}$. Comment ne pas percevoir dans ce réquisitoire une dénégation totale de cette conscience populaire collective. Les mots choisis par le procureur traduisent un sens profond et renvoient à des attitudes non-réfléchies qui obéissent à un instinct ou à une position irresponsable, et pas uniquement dans le sens d'une non-responsabilité au regard de la loi. On peut donc, en quelque sorte, les excuser, puisqu'ils ne savaient pas ce qu'ils faisaient. Il s'agit presque d'un aveu du procureur. Non pas un aveu d'impuissance, mais plutôt une confession publique où la volonté de bafouer ces hommes et ces femmes est réelle, puisque la Cour des Aides leur refuse un procès. En pardonnant, en ne voulant pas inculper un si grand nombre de personnes, la Cour des Aides refuse ce droit à la parole au peuple ${ }^{64}$.

Pendant près de dix jours, du 9 au 17 juillet 1789, les barrières de la capitale sont en proie aux incidents. Les dévastations se produisent dans les bâtiments, accompagnées quelquefois de pillage, avant qu'un grand nombre de bureaux soient incendiés entre le 11 et le 14 juillet ${ }^{65}$. Les barrières attaquées ne sont pas isolées dans l'espace. Elles forment la quasitotalité des lieux où se perçoivent les impôts. Une quarantaine de bureaux sur les cinquante-quatre en 1789 connaissent des dégradations ${ }^{66}$. Ce ne sont plus des émotions au sens d'un affrontement désordonné, passager et sans lendemain. Cependant, le terme d'affrontement n'a pas vraiment sa place, étant donné qu'il n'y a pas eu choc entre des adversaires ou ennemis. L'attaque aux barrières se déroule unilatéralement, l'opposition est inexistante. La volonté de la foule, au vu du contexte insurrectionnel, est une remise en cause de l'ordre établi.

On ne peut envisager ces actions uniquement comme des agissements hystériques et collectifs d'une foule qui s'attache à obtenir seulement

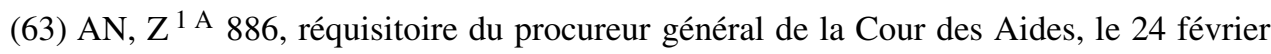
1790. Il n'est pas possible de développer cette approche ; retenons seulement que la procédure engagée contre les quatre-vingt individus se solde par l'arrestation et l'emprisonnement de douze personnes en juin 1790 (dont Musquinet déjà évoqué), considérés comme les meneurs de l'incendie. Mais devant l'hostilité grandissante de la population, l'Assemblée nationale, sollicitée par la Cour des Aides, se résout à abandonner toute poursuite à l'encontre des prévenus, avant de les relâcher au début du mois de juillet 1790 .

(64) Sur le regard que les élites intellectuelles portent sur le peuple, on renvoie à Deborah COHEN, La nature du peuple, les formes de l'imaginaire social (XVIII $-X X I^{e}$ siècles), Seyssel, Champ Vallon, 2010

(65) Dans de trop nombreux ouvrages historiques, seules les dates des 12 et 13 juillet sont retenues comme étant celles où les barrières furent incendiées.

(66) $\mathrm{AN}, \mathrm{Q}^{1} 1101$; 54 bâtiments sont déjà construits, le 55 ème , celui de Poissonnière n'a pas encore été édifié. 17 bureaux furent véritablement épargnés par le feu, essentiellement situés sur la partie septentrionale, de la barrière Blanche jusqu'à celle de Montreuil. 
une baisse du prix du vin ${ }^{67}$. L'objectif est clairement mis en évidence : supprimer les droits d'entrée. Ce nouvel ordre qui se met en place doit compter et composer avec le peuple. Si la forteresse a pu être prise, si la nouvelle municipalité parisienne s'empare du pouvoir, c'est parce que les événements, en rapport direct avec l'incendie des barrières, préparent la chute de la forteresse. Préparé, dans la mesure où avant même de connaître le 12 juillet le renvoi de Necker, d'autres Parisiens, à la périphérie, commencent à agir. Pas uniquement pour leur liberté, mais tout du moins pour montrer au pouvoir que le fameux mur élevé à si grands frais par la Ferme et avec l'accord explicite de la royauté, et sans avoir consulté la population, ne peut exister sans l'approbation du peuple. Le tiers état n'est plus seulement une idée abstraite. Ces hommes et ces femmes donnent un sens à ce qu'ils accomplissent. Ils font bloc, unis contre un adversaire commun et multiple. Ces êtres s'expriment par la voix, les cris, les gestes, la fureur, la violence. La manifestation de leurs pensées, l'expression de leurs actes s'insèrent durablement dans le cycle d'un changement durable et réel.

Certes, les deux événements, l'attaque des barrières et la prise de la Bastille, ne sont pas liés directement, et ceux qui se portent vers la prison royale ne sont peut-être pas ceux qui saccagent les barrières aux portes de Paris. Cependant, il n'est pas possible d'ignorer cette concomitance indirecte qui permet de libérer les esprits, les forces, et aller de l'avant. En ce sens, la «prise » des barrières ne doit plus être négligée.

\section{Annexe}

J'ai fait le choix d'indiquer sur la carte « La prise des barrières en $1789 »$ toutes les attaques contre les barrières de l'octroi y compris les bureaux des ports et quais qui dépendent de la Ferme. Lorsqu'il n'y a pas d'indication de date, cela signifie que les barrières ne furent pas assaillies ou que l'information directe est inexistante. C'est le cas pour les barrières de Courcelles et celle du Trône par exemple. Les dates indiquent les assauts sans distinguer spécifiquement la nature des dégâts (barrières saccagées

(67) Rappelons que cette foule ne correspond pas à la vision qu'avait Taine pour qui, cette multitude représente la « lie de la société [...] car ce que veut « la populace », c'est le « vin à bon marché »; Hippolyte TAINE, Les origines de la France contemporaine, tome 2, l'anarchie, Paris, Robert Laffont, 1986, p. 65. 
ou incendiées) ${ }^{68}$. Les tranches horaires ont été simplifiées : matin (8h$12 \mathrm{~h})$; après-midi (12h-18h) ; soir (18h-22h) ; nuit (22h-6h). La carte « Les barrières attaquées en juillet 1789 au nord de Paris » précise les assauts dans cette partie de la ville. Les deux cartes ont été élaborées à partir des procès-verbaux des employés de la Ferme et leurs témoignages lors de la procédure judiciaire menée par la Cour des Aides en 1790 (AN, Z1A 886).

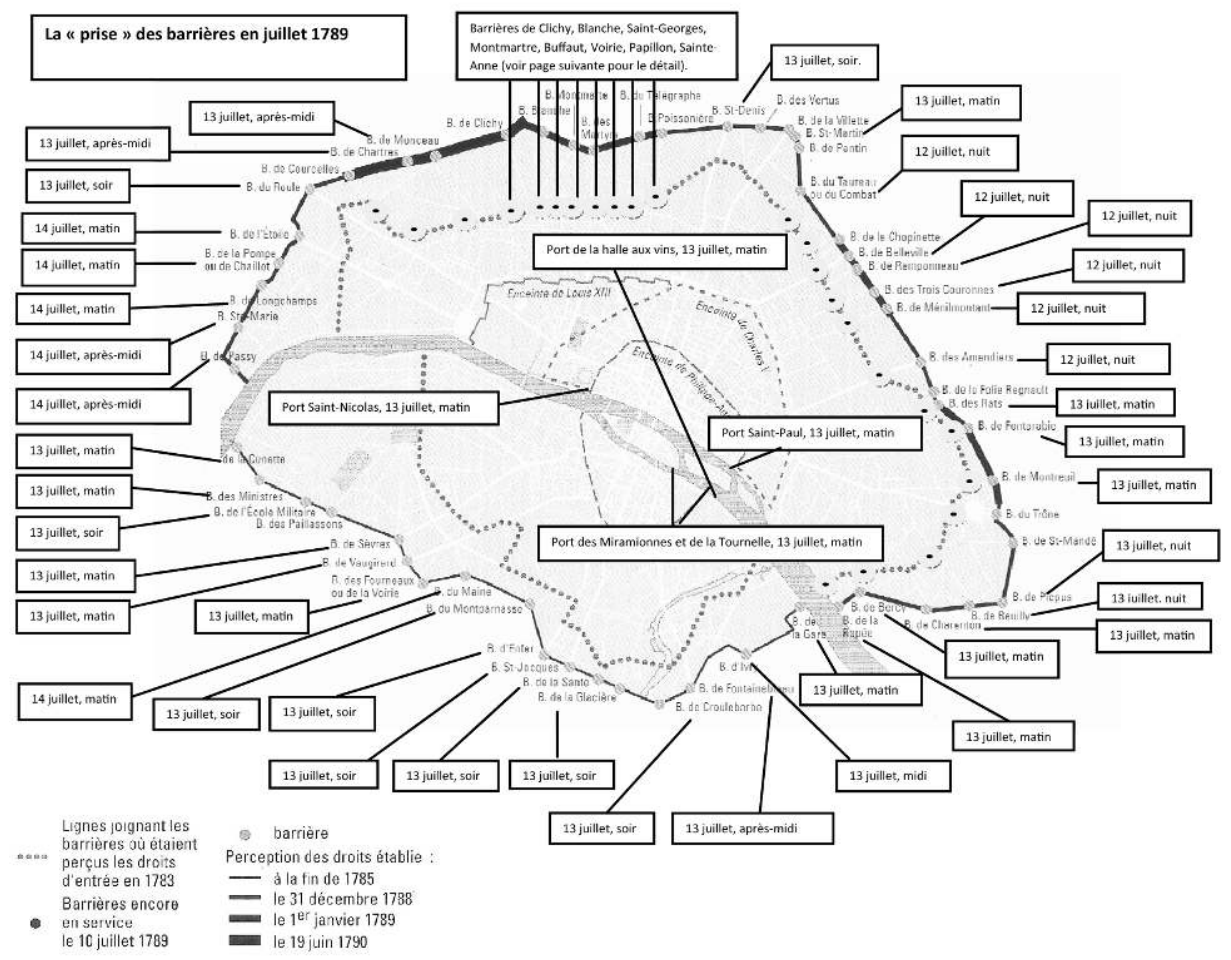

Figure 1. La prise des barrières en juillet 1789.

(68) La carte est établie d'après Émile DuCOUdRAY, Raymonde MONNIER, Daniel RochE (dir.), Atlas de la Révolution française, Paris, volume 11, Ecole des Hautes Etudes en Sciences Sociales, 2000. 


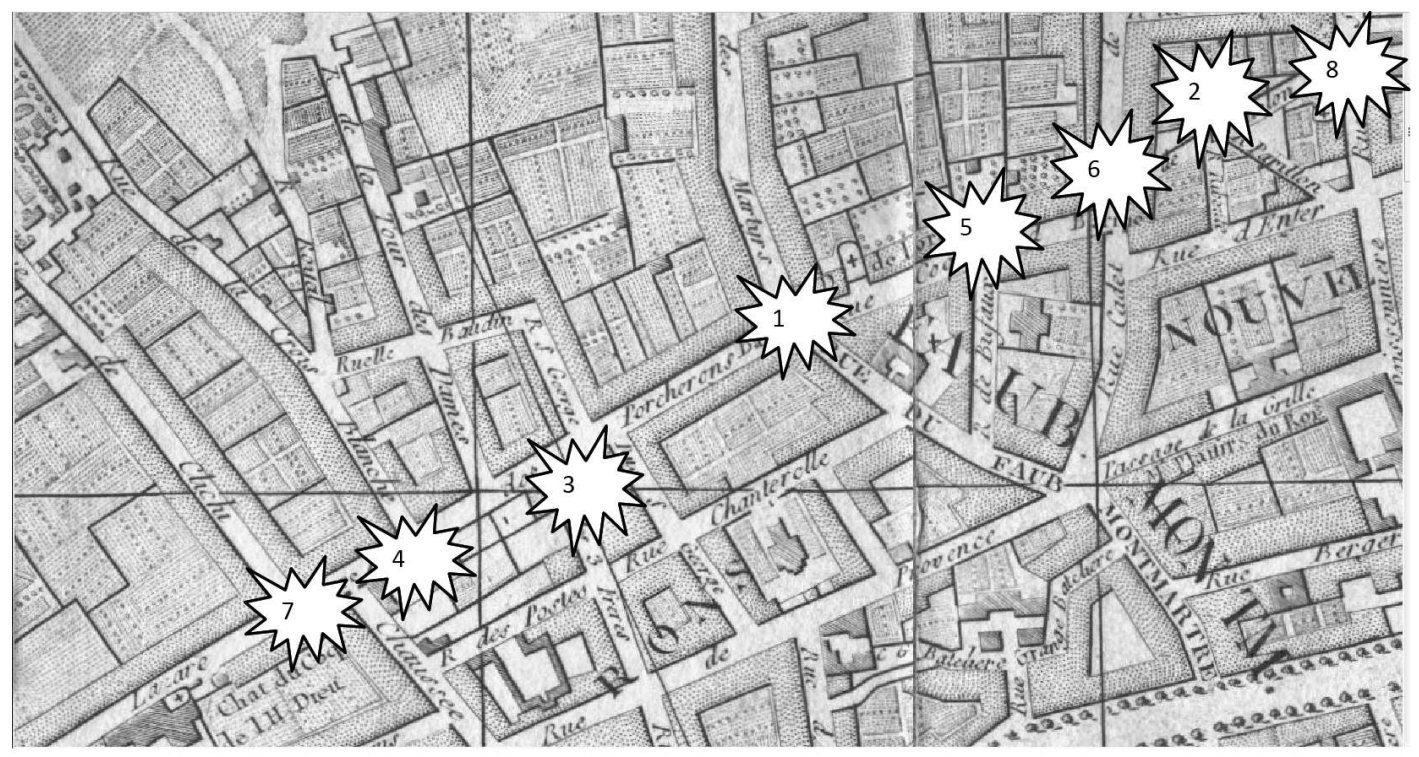

D’après Brion de la Tour, Nouveau plan de Paris, (plan partiel au nord), 1787.

$\begin{array}{ll}\text { 1. b. Montmartre (nuit du 9-10 juillet) } & \text { 2. b. Papillon (10 juillet, } 21 \mathrm{~h} \text { ) }\end{array}$

4. b. Blanche (11 juillet, $21 \mathrm{~h}$ )

5. b. Buffaut (11 juillet, $22 \mathrm{~h}$ )

8. b. Sainte-Anne (12 juillet, $21 \mathrm{~h}$ )

3. b. Saint-Georges et Trois frères (10 juillet, 23h)

7. b. Clichy (12 juillet à $16 \mathrm{~h}$ )

6. b. Voirie (12 juillet, $12 \mathrm{~h}$ )

Figure 2. Les barrières attaquées en juillet 1789 au nord de Paris (échelle : $1 \mathrm{~cm}=50 \mathrm{~m}$ )

Momcilo MARKOVIC

Doctorant à l'IHRF-université Paris 1

5 cité Trévise, 75009 Paris

momcilo.markovic@orange.fr 